\title{
Identification of QTL Combinations that Cause Spikelet Sterility in Rice Derived from Interspecific Crosses
}

\author{
Chang-Min Lee ${ }^{1}$, Jung-Pil Suh', Hyun-Su Park', Man-Kee Baek' , O-Young Jeong ${ }^{1}$, Song-Joong Yun², \\ Young-Chan Cho ${ }^{1}$ and Suk-Man $\mathrm{Kim}^{1,3^{*}}$ (1)
}

\begin{abstract}
Background: The exploitation of useful genes through interspecific and intersubspecific crosses has been an important strategy for the genetic improvement of rice. Postzygotic reproductive isolation routinely occurs to hinder the growth of pollen or embryo sacs during the reproductive development of the wide crosses.

Result: In this study, we investigated the genetic relationship between the hybrid breakdown of the population and transferred resistance genes derived from wide crosses using a near-isogenic population composed of 225 lines. Five loci (qSS12, qSS8, qSS11, ePS6-1, and ePS6-2) associated with spikelet fertility (SF) were identified by QTL and epistatic analysis, and two out of five epistasis interactions were found between the three QTLs (qSS12, qSS8 and qSS11) and background marker loci (ePS6-1 and ePS6-2) on chromosome 6. The results of the QTL combinations suggested a genetic model that explains most of the interactions between spikelet fertility and the detected loci with positive or negative effects. Moreover, the major-effect QTLS, qSS12 and qSS8, which exhibited additive gene effects, were narrowed down to 82- and 200-kb regions on chromosomes 12 and 8, respectively. Of the 13 ORFs present in the target regions, Os12g0589400 and Os12g0589898 for qSS12 and OS8g0298700 for qSS8 induced significantly different expression levels of the candidate genes in rice at the young panicle stage.
\end{abstract}

Conclusion: The results will be useful for obtaining a further understanding of the mechanism causing the hybrid breakdown of a wide cross and will provide new information for developing rice cultivars with wide compatibility.

Keywords: Spikelet fertility, Spikelet sterility, QTL, Interspecies cross, Hybrid breakdown, Rice

\section{Background}

Interspecific crosses have been continuously performed as effective counterplans to overcome the limited genetic diversity of cultivated rice (Tanksley and McCouch 1997). The introgression of useful genes or the emergence of new biotypes via recombination from various sources positively affect adaptation to the environment and sustain the potential for sustained genetic improvement over the long term. However, hybrid sterility, a postzygotic

\footnotetext{
${ }^{*}$ Correspondence: s_kim@knu.ac.kr

${ }^{1}$ Crop Breeding Division, National Institute of Crop Science, Rural Development Administration, Wanju, Republic of Korea

Full list of author information is available at the end of the article
}

reproductive barrier, is quite common in hybrid plants, which cannot produce fertile pollen or embryo sacs during reproductive development (Fang et al. 2019; Ouyang 2019). Thus, the enlargement of a wide compatibility ability using molecular technology could be considered breaking the reproductive barrier. Using this strategy in rice breeding will lead to the utilization of rice and extension of the rice cultivation area, which will prevent obstacles due to environmental or genetic factors.

Plant species, including rice, are isolated by various types of reproductive barriers according to their life cycle, which prevents individuals of genetically diverged groups from mating, surviving or producing fertile 
offspring (Widmer et al. 2009; Rieseberg and Blackman 2010; Ouyang 2019). Postzygotic reproductive isolation (RI) occurring after mating is known to drive speciation and maintain species identity, which blocks or reduces the gene flow among species (Kubo et al. 2016). A common form of postzygotic RI appears to be abnormal in offspring generations, as demonstrated by hybrid breakdown/weakness, hybrid sterility, and necrosis in the $F_{1}$, $\mathrm{F}_{2}$, or backcross generations (Jiang et al. 2008; Yamamoto et al. 2010; Ichitani et al. 2012; Ouyang and Zhang 2013). The syndrome is referred to as hybrid incompatibility proposed by the theory known as the "Bateson-Dobzhansky-Muller model" (Xie et al. 2019). Among the subtypes of postzygotic RI, hybrid necrosis and hybrid sterility have been recognized in $F_{1}$ hybrids, whereas hybrid breakdown occurs in the $F_{2}$ or later generations through a sterile or weakness phenotype (Yamamoto et al. 2010). To date, molecular genetic studies have revealed more than 50 loci that cause the fertility of inter(sub)specific crosses in rice, and these loci include loci with major effects and quantitative trait loci (QTL) with minor effects (Ouyang and Zhang 2013; Fang et al. 2019).

S5, S7, and HSA1 have been cloned and characterized with regard to hybrid female sterility, and $S a, S c$, and qHMS7 have also been characterized at the molecular level regarding male sterility. Hybrid sterility has been explained by two major genetic models: a one-locus allelic interaction model and a two-locus model (duplicate gametophytic lethal model) (Ouyang and Zhang 2013; Xie et al. 2019). In addition, sterility is mainly caused by the effect of multiple genes, which results in a very low pollen count and very low spikelet fertility, and sterility depends on a function of male gamete fertility, female gamete fertility, and affinity between the uniting male and female gametes (Song et al. 2005; Kubo et al. 2008). Additionally, there has been a study that crossincompatibility in $\mathrm{F}_{1}$ hybrid may be associated with interspecific cross or genes involved in the immune response (Bomblies and Weigel 2007; Kubo et al. 2008). Epistatic interactions are considered to contribute substantially to variations in spikelet fertility (SF) in hybrid progeny. Hence, a better understanding of the genetic mechanism responsible for the rice incompatibility should also be identified and characterized precisely despite these complexities or low heritability.

In this study, we performed a QTL analysis to establish the relationship between SF and resistance genes (R-genes) in the near-isogenic line (NIL) population. Using the NIL population with allelic fragments obtained by inter(sub)specific crossing, we identified five loci ( $q S S 12, q S S 8$, qSS11, ePS6-1, and ePS6-2) associated with SF and suggested a genetic model that explains the interactions within factors with positive or negative effects.
The results showed that one combination $(S S 12+$ SS8) described $50 \%$ of the observed SF, and other interactions, including epistatic factors (ePS6-1 and ePS6-2), were responsible for the remaining SF. The analysis revealed the genetic basis of SF and will contribute to the development of intermediate parents or cultivars with a wide compatibility line using the favorite genes of distantly related species in our japonica rice breeding programs.

\section{Results \\ Evaluation of SF Using the NIL Population Development of the Population}

Gene-pyramided line (GPL; a backcrossed line with the genetic background of Jinbu) is a NIL with the japonica background obtained from a marker-assisted backcross (MAB) based on an inter(sub)specific cross. Despite its advantage of multiple resistance, GPL is not optimal for practical use because it exhibits approximately $75 \%$ reduced fertility. Thus, we performed backcrossing again with Jinbu (japonica) to improve the SF (Additional file 1: Figure S1). Through foreground selection for each resistance gene ( $R$-gene), ten cross plants were selected and advanced to generate an $\mathrm{F}_{2}$ population. From $240 \mathrm{~F}_{2}$ individuals, 225 lines were subsequently developed by measuring the main agronomic traits and performing marker selection for the $R$-genes and then advanced by singleseed descent (SSD).

\section{Segregation Ratio of the SF}

The SF of the developed 225 NILs was evaluated. The degree of SF ranged widely from 10 to $100 \%$ in the population and did not appear to exhibit a normal distribution curve on the tested trait (Fig. 1). While major agronomic traits showed a normal distribution pattern within the range of the parents at the same stage without symptoms of poor growth or inviability (data not shown). The segregation of the SF was analyzed to understand the inheritance pattern (Table 1). According to the reference point $(\mathrm{RF})$, the segregation ratio was not fitting the radio of 1:2:1, but to fit 9:3:4 (F:M:S) at RF-I and RF-II ( $X^{2}$ for 9:3:4, $P<0.05)$. Because the SF of both parents was higher than $75 \%$, the inheritance pattern of the NIL population suggested that multiple genes and not a single gene would dominate the SF.

\section{Analysis of the Relationship Between the R-Genes and Spikelet Sterility (SS)}

To identify the cause of occurrence of SS in the population, the relationship between the SF values and the introgression of $R$-genes was analyzed using correlation coefficients and ANOVA. The analysis revealed that Bph18 only showed a weak positive correlation $(r=0.36)$ with SF, whereas other genes did not show any 


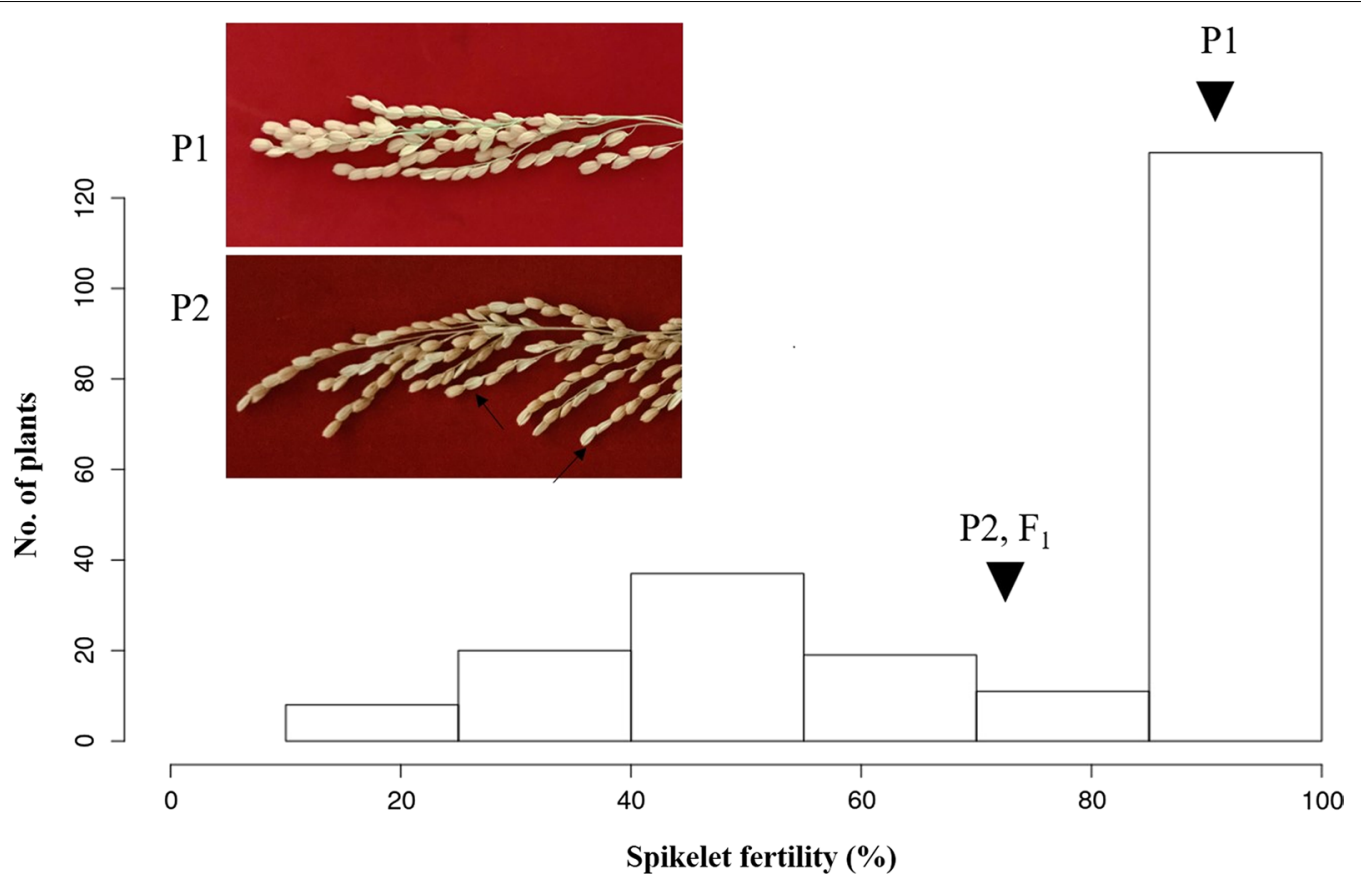

Fig. 1 Frequency distribution of spikelet fertility among the 225 tested lines. $F_{1}$ and the parent showed more than $75 \%$ spikelet fertility. P1 refers to Jinbu, and P2 is GPL

Table 1 Segregation ratio of spikelet fertility (SS) in the tested 225 lines

\begin{tabular}{|c|c|c|c|c|c|c|c|}
\hline \multirow[t]{2}{*}{ Reference point (RF) } & \multirow[t]{2}{*}{ Seg } & \multicolumn{4}{|c|}{ No. of plants } & \multicolumn{2}{|l|}{$X^{2}$ value } \\
\hline & & Fertile & Moderate & Sterile & Total & $1: 2: 1$ & 9:3:4 \\
\hline RF-I & $\mathrm{F}: \mathrm{M}: \mathrm{S}$ & 130 & 52 & 43 & 225 & $132.4^{* *}$ & $5.49^{\mathrm{ns}}$ \\
\hline RF-II & $F: M: S$ & 130 & 31 & 65 & 225 & $156.4^{* *}$ & $4.97^{\mathrm{ns}}$ \\
\hline RF-III & $F: M: S$ & 130 & 20 & 75 & 225 & $179.0^{* *}$ & $18.01^{* *}$ \\
\hline
\end{tabular}

Seg.: segregation of spikelet fertile classified into three classes: $\mathrm{F}$ (Fertile), $\mathrm{S}$ (Sterile), and $\mathrm{M}$ (Moderate fertile) according to reference point (RP) distinguish fertility from sterility. RF-I (F: M: S= over 85\%: 85-45\%: less than 45\%), RF-II (F: M: $S=85: 85-55: 55)$, RF-III (F: M: S=85: 85-65: 65)

${ }^{n s}$ Not significant

Significant levels: ${ }^{*} P<0.05$ and ${ }^{*} P<0.001$

correlation with the trait (Additional file 1: Figure S2). In addition, an ANOVA $\mathrm{F}$ test for the three $\mathrm{R}$-genes $\mathrm{Xa4}$, Pi40, and Bph18 was significant, providing no difference in interaction within the $R$-genes for SS in the population (Additional file 1: Table S1).

\section{Analysis of QTLs for SF}

QTL analysis was performed using a linkage map constructed using Kompetitive allele-specific PCR (KASP) marker sets (Additional file 2: Table S2). A total of 127 markers were eventually selected to be anchored on the 12 rice chromosomes (Additional file 1: Table S3). The linkage map showed an average distance of $8.62 \mathrm{cM}$ within the flanking markers. The QTL analysis identified a total of three QTLs associated with SS through inclusive composite interval mapping (ICIM) and an empirical threshold of LOD $>2.70$ (Table 2). The QTLs $q S S 8, q S S 11$, and $q S S 12$ on chromosomes 8, 11, and 12 were continuously detected for the experimental periods within the years 2018-2019 (Fig. 2). The QTLs revealed a negative influence on the fertility of the population. Of the two QTLs ( $q S S 12$ and qSS11) derived from GPL, qSS12, with LOD scores of 15.5 and 14.7 within 7312 . T4A (Bph18) and Kj12_061, was identified as the main QTL explaining $24.0 \%$ and $23.5 \%$ of the phenotypic variation $\left(R^{2}\right)$ in the ICIM analysis, respectively. Additionally, $q S S 8$ and $q S S 11$, with LODs of 7.5-8.6 and 3.7-4.0, respectively, were detected within the flanking markers KJ08_040-JH08_070 and KJ11_013-KJ11-015 on chromosomes 8 and 11 with $R^{2}$ values of $10.5-12.6$ and 
Table 2 QTLs associated with spikelet fertility of 225 lines detected by composite and interval mapping

\begin{tabular}{|c|c|c|c|c|c|c|c|c|c|}
\hline \multirow[t]{2}{*}{ QTLs } & \multirow[t]{2}{*}{ Chr } & \multicolumn{4}{|l|}{2018} & \multicolumn{4}{|l|}{2019} \\
\hline & & Franking-markers & LOD & PVE (\%) & Add & Franking-markers & LOD & PVE (\%) & Add \\
\hline qSS8 & 8 & KJ08_40-KJ08_70 & 7.5 & 10.5 & -9.6 & KJ08_40-KJ08_70 & 8.6 & 12.6 & -10.9 \\
\hline qSS11 & 11 & KJ11_13-KJ11_15 & 3.7 & 4.7 & 7.0 & KJ11_13-KJ11_15 & 4.0 & 5.2 & 7.8 \\
\hline qSS12 & 12 & 7312.T4A-KJ11_61 & 15.5 & 24.0 & 15.4 & 7312.T4A-KJ11_61 & 14.7 & 23.5 & 15.8 \\
\hline
\end{tabular}

PVE (\%): Percentage of phenotypic variation explained by the QTL, Add: additive effect

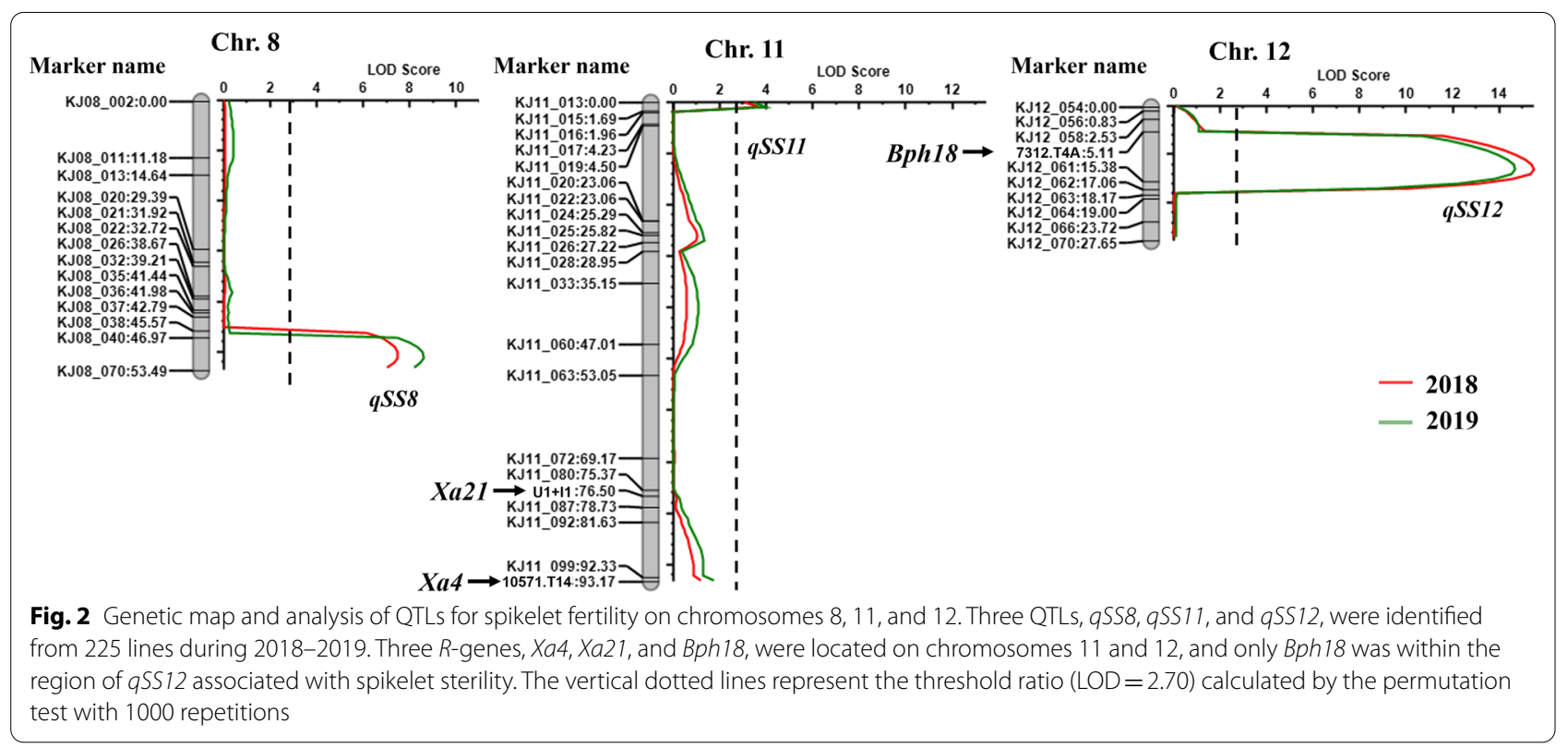

$4.7-5.2 \%$, respectively. The allele of $q S S 8$ was derived from Jinbu, and $q S S 11$, similar to $q S S 12$, was obtained from GPL.

\section{Confirmation of the Effect of the QTL Combinations on SS}

To verify the influence of the three QTLs for SS on the population, the combination effects were assessed by comparing the difference in the values to the number of QTLs detected in this study (Additional file 1: Figure S3). The results showed that the existence of QTL(s) divided the mean values of the SF of the tested QTL combinations into fertility and sterility. However, we also investigated the segregation pattern of the SF values in two cases ( $q S S 12$ and $q S S 8+q S S 11)$. The QTL combination composed of eight allelic types was explained by the allele type of the QTLs (Table 3). All the genotypes involved in TYPE_A belonging to the homozygote $q S S 12^{-}$inbu exhibited high SF, regardless of the presence of the others. In TYPE_B without $q S S 12^{-}{ }^{\text {linbu }}$, all lines, including $q S F 8^{-{ }^{\text {inbu }}}$ in genotypes I (Type B-I) and II (Type B-II), showed high SS, whereas lines of genotypes III (Type B-III) and VI (TYPE
Table 3 Spikelet fertility of the tested lines using the combinations of the three QTLs based on allele types detected from the parents

\begin{tabular}{|c|c|c|}
\hline \multirow[t]{2}{*}{ Genotypes } & \multicolumn{2}{|c|}{ Allele type of $q S S 12$} \\
\hline & $\begin{array}{l}\text { TYPE A ( } q S S 12- \\
\text { Jinbu) }\end{array}$ & TYPE B ( $\left.q S S 12^{-G P L}\right)$ \\
\hline qSS8-Jinbu/qSS11_GPL & Fertile & Sterile \\
\hline II. $q S S 8^{-}{ }^{\text {Jinbu }} / q S S 11_{-}^{-J i n b u}$ & Fertile & Sterile \\
\hline III. qSS8_GPL/qSS11__linbu & Fertile & Sterile and fertile \\
\hline VI. qSS8-GPL/qSS11_GPL & Fertile & Sterile and fertile \\
\hline
\end{tabular}

B-VI) presented segregation patterns for SF ranging from 10 to $90 \%$. The results explained that all the

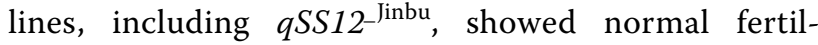
ity, whereas all the lines with the QTL combination $\left(q S S 12^{-\mathrm{GPL}}+q S S 8^{-\mathrm{Jinbu}}\right)$ continuously presented high SS in this population. In addition, given the segregation of SF observed, it could be assumed that other factors that 
had not yet been identified were involved epistatically, resulting in the occurrence of SS in the population.

\section{QTL-Background Loci Interaction for SS}

An epistatic analysis was performed to identify the phenomenon of segregation in TYPE B_III and TYPE B_VI. Based on the analysis, digenic interactions were detected at five chromosomal positions with either negative or positive influences on SS (Additional file 1: Table S4). Two out of five epistasis interactions were found between the three QTLs (qSS12, qSS8 and qSS11) and background marker loci on chromosome 6; the loci were renamed $e P S 6-1$ and $e P S 6-2$. The others were previously detected QTL-QTL interactions; a negative effect for SS was found within qSS8-qSS12 and qSS8-qSS11, and a positive interaction was found in others. The phenotypic variation explained (PVE) by the epistatic QTLs ranged from 5.37 to $15.98 \%$ in the analysis. Moreover, qSS8 on chromosome 8 exhibited digenic interactions with all loci with the exception of ePS6-1 on chromosome 6.

\section{Genetic Models for SF in the Lines}

Using the results of the QTL and epistasis analyses, we constructed a genetic model to explain SS (Fig. 3). The procedure was performed by adding high-effect QTLs one by one in order. If a particular QTL combination exhibits a pattern of separating SF, it was added to the analysis, whether it matches the phenotype was assessed. Segregation issues were observed three times in the analysis, and the combinations were classified into CsgI, Csg-II, and Csg-III in order. The combinations Csg-II $\left(q S S 12^{-}{ }^{\mathrm{GPL}}+q S S 8^{-}{ }^{\mathrm{GPL}}\right)$ were divided into four genotypes based on additional loci, $q S S 11$ and $e P S 6-2$. In the combinations obtained by adding $q S S 11^{-{ }^{\mathbf{G P L}}}$ to Csg-II, the SF phenotype was semi-fertile in the presence of ePS6$2^{-\mathrm{GPL}^{-}}$and sterile in the presence of $e P S 6-2^{-\mathrm{Iinbu}}$, but in the case of Csg-II $+q S S 11^{- \text {Jinbu }}$, the phenotype was fertile in the presence of $e P S 6-2^{-G P L}$. Moreover, the combination Csg-III $\left(\mathrm{Csg}-\mathrm{II}+q S S 11^{\text {Jinbu }}+e P S 6-2^{\text {- }}{ }^{\text {linbu }}\right)$ only showed a segregated pattern for SF. In this case, ePS6-1 acted additively on qSS12 to serve as a key factor in determining whether the phenotype was fertile or sterile. With the addition of $e P S 6-1^{-\mathrm{GPL}}$ to Csg-III, the SF phenotype was sterile, and the SF in the absence of $e P S 6-1^{-}{ }^{\mathrm{GPL}}$ was normal. The interaction of two QTLs, qSS12 and qSS8, induced hybrid breakdown, which explained $50 \%$ of the observed SF in the population. Another 50\% of the SF was explained by the interaction between other factors (qSS11, ePS6-1, and ePS6-2) and both QTLs.

\section{Physical Positioning of the Detected QTLs}

The target regions of $q S S 12$ and $q S S 8$ were narrowed down using each plant group selected as the recombinant and delimited within approximately $82-\mathrm{Kbp}$ and 200-Kbp segments flanked by cleaved amplified polymorphic sequences (CAPS) and derived cleaved amplified polymorphic sequences (dCAPS) marker sets (Fig. 4). The recombinant lines showing discordance between phenotype and genotype were used to dissect the flanking region of the main QTL qSS12, which was found to be anchored from 22.8 to $25.2 \mathrm{Mbp}$ on chromosome 12. A total of five recombinant plants selected by the flanking markers 7312.T4A (Bph18) and KJ12_061 were used to narrow down the target region (Fig. 4A). For dissection of the target QTLs, additional markers were newly designed according to the data from the whole-genome resequencing (WGR) analysis of the parents (Additional file 2: Table S5). Following the same procedures, fine mapping for the QTL qSS8 was performed using the six recombinants (Fig. $4 \mathrm{~B}$ and Additional file 2: Table S6).

Based on the results, the target region was narrowed to approximately 82 -Kbp segments delimited by ST24_38 and ST24_II_2. Six ORFs based on Os-Nippobare-Reference-IRGSP-1.0 were identified in the target region (Additional file 2: Table S7). The position of $q S S 8$ was also narrowed down to identify the association with SS. The target region was flanked from approximately $200-\mathrm{Kbp}$ segments delimited by ST8_dcII_10 and ST8_dcIII_19, and 7 ORFs were identified in the target region (Additional file 2: Table S7). From the fine-mapping results, we placed six and 13 ORF candidates in target regions for $q S S 12$ and $q S S 8$, respectively.

\section{Expression of Genes by qPCR}

We designed new primer sets to analyze the expression levels of 13 ORFs identified in the target regions on chromosomes 8 and 12 based on the Rice Annotation Project Database (RAP-DB, http://rapdb.dna. affrc.go.jp/). Of the six candidate genes for qSS12, two genes, Os12g0589400 and Os12g0589898, exhibited upregulated expression levels in only a panicle of GPL and were expressed at significantly higher levels in GPL than in Jinbu (Fig. 5A). An increase in the expression levels of the genes was observed in GPL at 0 days after heading (DAH), and the measured expression level was 11 times the concentration at 5 days before heading $(\mathrm{DBH})$ and almost returned to its original level at 30 $\mathrm{DAH}$. In the case of $q S S 8$, however, the expression level of Os08g0298700 was upregulated in Jinbu at $0 \mathrm{DAH}$ (Fig. 5B) to a sixfold higher concentration compared with that at $5 \mathrm{DBH}$ and $30 \mathrm{DAH}$. The remaining ORFs 


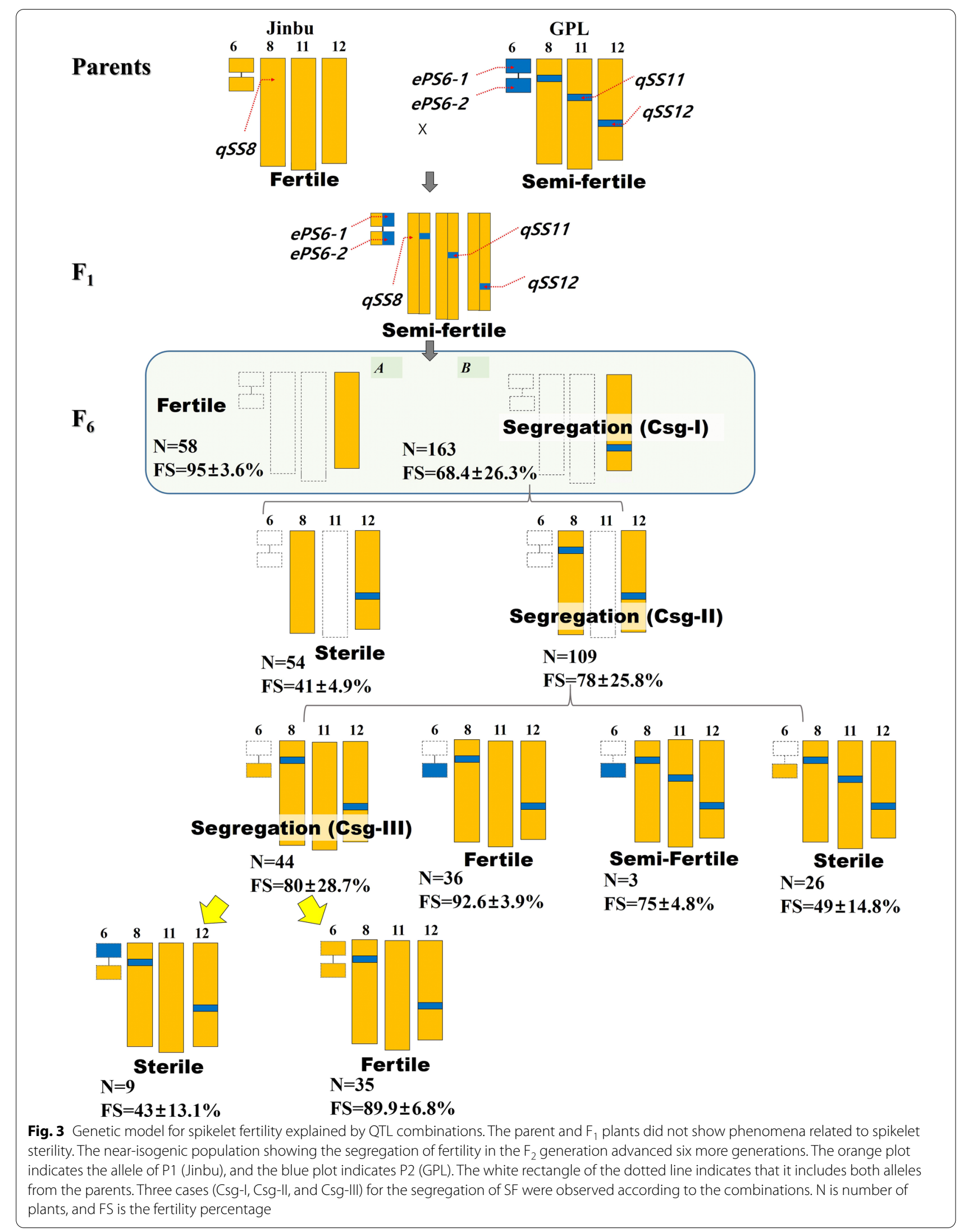



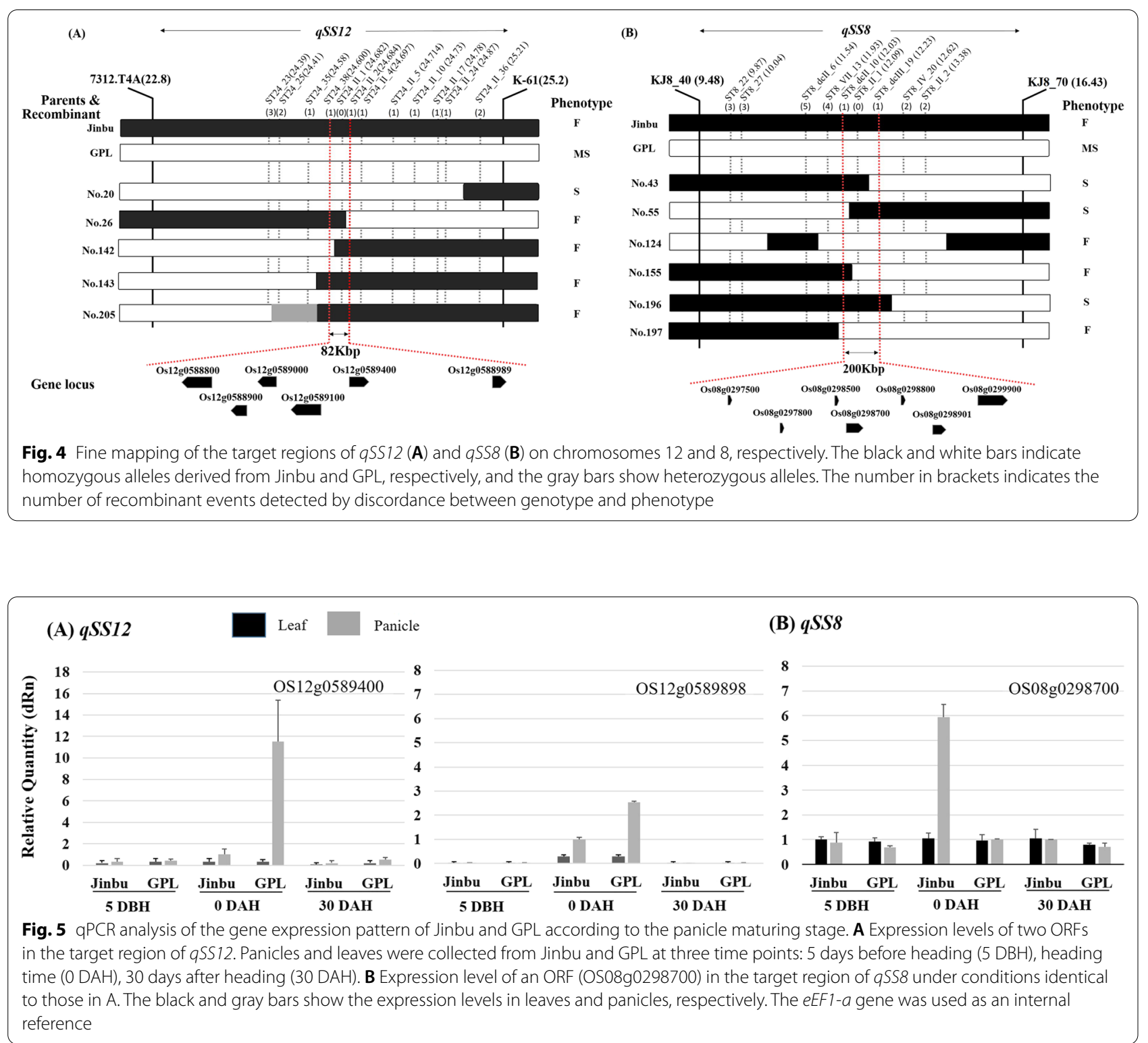

revealed no significant difference between the parents. In particular, the qPCR analysis using lines with both QTLs qSS12 and qSS8 showed that both genes were expressed independently with no interaction at the RNA level (data not shown).

\section{Discussion}

The inter(sub)specific cross is often considered for exploiting strong heterosis or overcoming the limitations of the genetic background, particularly in japonica breeding programs against various stresses (Ouyang 2019). However, the reduced fertility or weakness observed in the cross serves as a barrier to the further utilization of a wide range of subjected advantages.
In this study, we performed a QTL analysis to identify the cause of SS observed in 225 NILs derivatized from a cross between the japonica cultivar Jinbu and the introgression line (IL) GPL with a japonica genetic background (Additional file 1: Figure S1). In a preliminary study, $F_{1}$ plants derived from a cross between Jinbu and GPL were obtained to improve the SF of the IL as part of a breeding program, and the resulting plants exhibited approximately $75 \% \mathrm{SF}$ under field conditions. At the $\mathrm{F}_{1}$ stage, no poor characteristic phenotype, called postzygotic isolation, was observed, whereas wide-ranging segregation manifested in the $\mathrm{F}_{2}$ generation through the sterility phenotype. This hybrid breakdown is one of the postzygotic isolations observed in $F_{2}$ or later generations 
produced by interspecific crosses (Yamamoto et al. 2010). In this study, we transferred five $R$-genes into the japonica cultivar Jinbu to develop multiple resistant cultivars by interspecific crossing; however, we observed hybrid breakdown in the segregating generation. In fact, hybrid breakdown causing high sterility shows typical polygenic inheritance; hence, it is conceivable that the interaction among the polygenes is related to the sterility mechanism (Kubo et al. 2008).

To confirm whether the issue was caused by pyramiding $R$-genes, statistical analyses were performed using the NILs developed in this study (Additional file 1: Figure S1 and Table S1). The results revealed that SS was not fully interpreted by a single gene or combinations of genes, with the exception of Bph18 on chromosome 12, which showed a weak positive correlation $(r=0.36)$ with the trait. Bph18 conferring resistance to brown plant hopper $(\mathrm{BPH})$ encodes the NBS-NBS-LRR protein in the endomembranes in a cell, and the gene might recognize $\mathrm{BPH}$ invasion at endomembranes in phloem cells (Ji et al. 2016). According to previous reports, NBS-LRR triggers the immune response signal in response to pathogen attack and is involved in an effector-triggered immunity system in which the NBS-LRR recognizes avirulence proteins either directly or indirectly (Chisholm et al. 2006; DeYoung and Innes 2006).

Through linkage analysis, we identified QTLs associated with SS, which was assumed to be the cause of the deleterious interaction with the transferred $R$-gene Bph18. In the analysis, three QTLs, qSS8, qSS11, and qSS12, were identified on chromosomes 8,11 and 12, respectively (Table 2 and Fig. 2). Based on the analysis, all QTLs associated with SS had LOD values of 25.8-26.6 and explained $35.9-38.4 \%$ of the phenotypic variation within two years by CIM. Further epistatic QTL analysis allowed us to identify two additional loci (ePS6-1 $1^{\mathrm{GPL}}$ and $e P S 6-2^{-G P L}$ ) on chromosome 6 . To date, hybrid sterility or weakness has mainly been thought to be caused by onelocus allelic interactions at a single locus and a two-locus model (Chen et al. 2008; Long et al. 2008; Kubo et al. 2016; Shen et al. 2017; Xie et al. 2017; Koide et al. 2018). In addition, the accumulated effect of multiple loci has been widely reported to be associated with weakness or SF in rice (Wang et al. 1998; Yamamoto et al. 2007; Kubo et al. 2011). In fact, the interaction among polygenes is related to the sterility mechanism and exhibits typical polygenic inheritance (Zhao et al. 2007; Kubo et al. 2008). Song et al. (2005) identified six QTLs on chromosomes 5, 6,8 , and 12 that control the SF of intersubspecific hybrids of rice. Kubo and Yoshimura (2005) reported three loci designated $h s a 1, h s a 2$, and $h s a 3$ on chromosomes 12, 8 and 9 that are involved in hybrid breakdown by female gametes in a japonica-indica cross.
Based on the analysis of QTL combinations, we established a genetic model that explains the interaction among five loci ( $q S S 12^{-\mathrm{GPL}}, q S S 8^{-\mathrm{Jinbu}}, q S S 11^{-\mathrm{GPL}}$, $e P S 6-1$, and $e P S 6-2)$ associated with SF. The combination composed of the two loci SS12 (qSS12-GPL) and SS8 $\left(q S S 8^{-}{ }^{\text {inbu }}\right)$ exerted the most dominant effects on SF in the analysis. $q S S 12^{-\mathrm{GPL}}$, which is named SS12 on chromosome 12, is the main QTL associated with SF. Considering the fertility level of the parent GPL, $q S S 8^{-}{ }^{\mathrm{GPL}}$ appears to inhibit or hinder the role of $S S 12$, or $q S S 8_{-}^{\text {Jinbu }}$ appears to strengthen the role in triggering SF. The reason for this finding is that all the plants in which $\mathrm{qSS}^{-}{ }^{\mathrm{GPL}}$ was replaced by $q S S 8^{- \text {Jinbu }}$, including the combination $\left(q S S 12^{-{ }^{G P L}}+q S S 8^{- \text {Sinbu }}\right)$, showed severe sterility (Table 3$)$. Despite their high involvement in sterility, the combination explained only approximately $50 \%$ of the SF in the population. The remaining SF could be explained by ePS6-1 and 6-2 on chromosome 6 with more complex interactions. ePS6-2 interacted with $q S S 8^{-}{ }^{\mathrm{GPL}}$ and was negatively affected by SS, whereas $e P S 6-1$ interacted with $q S S 12^{-\mathrm{GPL}}$ and was positively involved in SS. The case of qSS11- ${ }^{\mathrm{GPL}}$ also showed an association with sterility, which indicated an additive effect on the tested trait. Based on the results, we could clearly explain most of the SF in the population using the five loci detected in this study. Moreover, the main QTL combination $(S S 12+S S 8)$ in this study tended to be similar to epistasis by $h s a 1$ and has2 (Kubo and Yoshimura 2005).

We performed fine mapping to each region of the main QTLs (qSS12 and qSS8) for SS in the population using WGR data. From the results we could delimited the targets within $82-\mathrm{Kbp}$ and $200-\mathrm{Kbp}$ segments on each chromosome. Contrary to our expectations, however, no known $R$-genes were identified. In conclusion, $B p h 18$ was not included in the range of $q S S 12$, and then the SS was not related to the autoimmune response by the $R$-genes introgression. It is simply a result of being transferred to epistasis together, causing SS during hybridization because of their close physical location.

To confirm the candidate gene(s) for SS12 and SS8, qPCR was conducted using primer sets developed based on the sequences in exons. For SS12, the expression levels of only two genes, Os12g0589400 and Os12g0589898, were significantly higher in GPL than in Jinbu, as determined by qPCR (Fig. 5A). Both ORFs encoded "Domain of unknown function (DUF1618 domain)-containing protein" and "Similar to 2-isopropylmalate synthase B" as putative functions, respectively. SS12 was identified at the position of $h s a 1$ containing HSAla and $b$, which is known as the hybrid sterility gene (Kubo et al. 2016). The genetic positions occupied by the two genes appear to be identical and performed functions negatively associated with the fertility of rice, even though both were 
derived from different allelic sources, i.e., SS12 and hsa1 originated from $O$. australiensis and $O$. sativa indica, respectively. Moreover, among the candidates for SS8, the expression level of only OS08g0298700 was upregulated in the panicle of Jinbu at $0 \mathrm{DAH}$ (Fig. 5B), and the ORF was known to encode "Similar to male sterility protein 2". However, the actual reason for SS determined in this study was far from the problem with pollen (Additional file 1: Figure S4). SS12 and SS8 were independently expressed in the plants with each corresponding allele, regardless of whether the plant's phenotype was fertile or sterile. This fact suggested that the two genes did not appear to affect each other's expression. Given the discrepancy between gene expression and phenotype, we hypothesize that SS could be caused by interactions within encoded proteins or by additive accumulation of each QTL effect.

\section{Conclusions}

In this study, we identified novel gene combinations controlling SS in a NIL population with a japonica genetic background derived from an interspecies cross. Based on the results, the combinations composed of the five loci were involved in SS either positively or negatively through interactions among the loci. Additionally, a genetic model explaining a set of complementary genes controlling hybrid breakdown in rice was suggested by the results from the QTL and epistatic analyses. The current results facilitate the establishment of compatible cultivars using PCR-based markers developed in this study. Furthermore, a functional analysis of the identified genes will be performed in a follow-up study. A mechanistic understanding of incompatibilities, such as hybrid breakdown caused by a wide cross, will help increase the availability of various useful genetic sources for rice breeding programs, and the approach performed in this study will be more effective for japonica cultivars with a relatively narrow genetic background.

\section{Materials and Methods}

\section{Plant Materials and Mapping Population}

The japonica cultivar Jinbu and the GPL were used as the parental cultivars for the mapping population. Jinbu is an early-maturing cultivar with cold tolerance and good grain quality. The other parent, GPL, has five different $R$-genes introduced by inter(sub)specific crosses (Additional file 1: Figure S5) (Suh et al. 2015). Three R-genes, $X a 4, x a 5$, and $X a 21$, for bacterial leaf blight resistance were introgressed from a donor derived from $O$. sativa subsp. Indica, and two R-genes, Pi40 and Bph18, for blast and BHP, respectively, were introduced by two lines, IR65482-4-136-2-2 and IR65482-7-216-1-2, which are resistance donors derived from $O$. australiensis.
To increase the SF of GPL, GPL was backcrossed again with Jinbu, and 225 NILs were developed using a single descent method. The population based on the japonica background was used to access the phenotypic data and to construct a molecular genetic map for identification of QTLs controlling SF.

\section{Evaluation of the SF Rate}

The population was cultivated and evaluated in an experimental field at the National Institute of Crop Sciences (NICS), RDA, Wanju, South Korea, from 2018 to 2019. For evaluation of the fertility rate, three panicles with fully ripened grains were collected from the plants, and the numbers of filled and unfilled spikelet seeds were counted. The fertility rate was estimated using the following formula: fertility rate=number of filled grains/total number of filled and unfilled grains (Kubo et al. 2016). To distinguish fertility and sterility, we needed to identify a RF for determining a criterion for SF in the population because the value of SF was continuously obtained from the phenotypic evaluation. After a fixed fertility level of over $85 \%$, the RF was divided into three classes based on moderate SF (MSF) levels: RF-I (S:M:F= less 45\%:45-85\%:over 85\%), RF-II (F:M:S= less than 55\%:55-85\%:over $85 \%$ ) and RF-III (F:M:S = less than 65\%:65-85\%:over $85 \%$ ).

\section{Genotyping and Linkage Mapping}

For QTL analysis, we used a total of 771 KASP markers developed to detect single nucleotide polymorphisms (SNPs) of Korean japonica rice varieties (Cheon et al. 2018). To confirm that the markers were anchored on each chromosome, some SNPs among the genotypic datasets were removed if biased segregation appeared within the SNPs at overlapping positions on the chromosomes. The selected KASP markers were used to construct a genetic linkage map using the linkage mapping software QTL IciMapping, version 4.1 (Meng et al. 2015).

\section{Data Analysis}

To determine the relationship between SF and the $R$-genes introduced into the correlation analysis, the corrplot package in $\mathrm{R}$ was used. Two-way ANOVAs were conducted to study the main effects of the five resistance markers and their interactions using $\mathrm{R}$ version 4.0.2. QTL analysis and epistatic mapping were conducted using conventional mapping for inclusive composite interval mapping for additive QTLs (ICIM-ADD) (Meng et al. 2015). Permutation tests with 1000 replicates $(P \leq 0.05)$ were applied to confirm the significant threshold values 
of the LOD scores for QTL detection (Churchill and Doerge 1994). The epistatic interactions between marker loci of RILs for SF were analyzed using the ICIM-EPI functions in QTL IciMapping version 4.1. A threshold LOD of 4.0 with probability values for entering variables (PIN) of 0.0001 was used to define the significant epistatic QTLs (Chattopadhyay et al. 2019).

\section{Development of DNA Markers and Whole-Genome Resequencing (WGR)}

For fine mapping, we designed new primer sets to narrow down the target region using the WGR data of the parents. Particularly for CAPS and dCAPS, specific restriction sites in the target region were detected using the marker design software RICE SNP-MINER. WGR was performed using an Illumina NovaSeq 6000 system (Illumina, USA) following the provided protocols for $2 \times 100$ sequencing. The DNA library was prepared according to the TruSeq Nano DNA library preparation protocol (Cat. No. FC-121-4001).

\section{qPCR Analysis}

The real-time PCR array was performed using an Exicycler $^{\mathrm{TM}} 384$ Real-Time Quantitative Thermal Block (Bioneer, Daejeon, Korea) with the following cycling parameters: 40 cycles of $95{ }^{\circ} \mathrm{C}$ for $5 \mathrm{~s}, 58{ }^{\circ} \mathrm{C}$ for $25 \mathrm{~s}$ and $72{ }^{\circ} \mathrm{C}$ for $30 \mathrm{~s}$. Data analysis was performed based on the relative quantitative method, and the $\Delta \Delta C T$ value was used to determine the relative fold change in expression. All the data were normalized to the expression level of the reference gene Os03g08010 (eEF1-a).

\section{Supplementary Information}

The online version contains supplementary material available at https://doi. org/10.1186/s12284-021-00540-6.

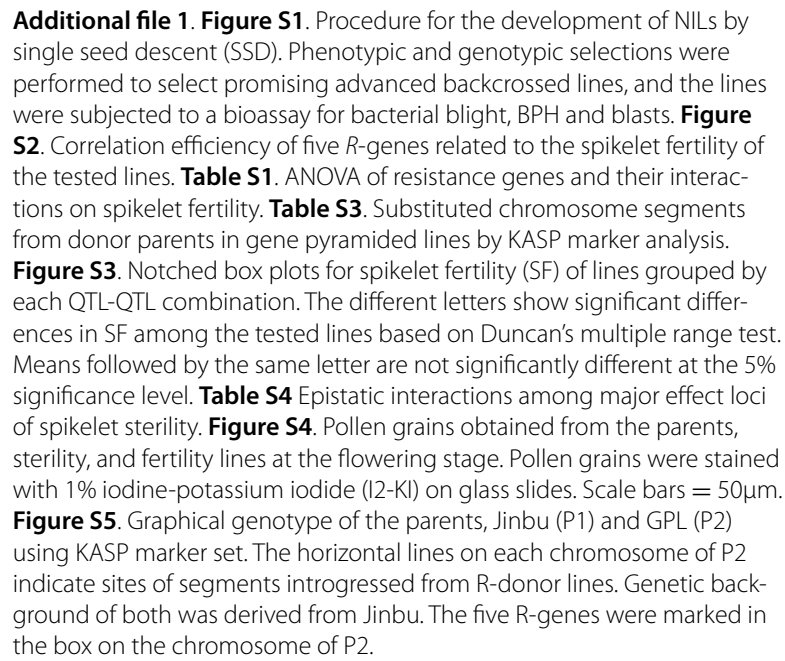

Additional file 1. Figure S1. Procedure for the development of NILs by single seed descent (SSD). Phenotypic and genotypic selections were performed to select promising advanced backcrossed lines, and the lines were subjected to a bioassay for bacterial blight, $\mathrm{BPH}$ and blasts. Figure S2. Correlation efficiency of five $R$-genes related to the spikelet fertility of the tested lines. Table S1. ANOVA of resistance genes and their interactions on spikelet fertility. Table S3. Substituted chromosome segments from donor parents in gene pyramided lines by KASP marker analysis. Figure S3. Notched box plots for spikelet fertility (SF) of lines grouped by each QTL-QTL combination. The different letters show significant differences in SF among the tested lines based on Duncan's multiple range test. Means followed by the same letter are not significantly different at the 5\% significance level. Table S4 Epistatic interactions among major effect loci of spikelet sterility. Figure S4. Pollen grains obtained from the parents, sterility, and fertility lines at the flowering stage. Pollen grains were stained with $1 \%$ iodine-potassium iodide $(\mathrm{I} 2-\mathrm{KI})$ on glass slides. Scale bars $=50 \mu \mathrm{m}$ Figure S5. Graphical genotype of the parents, Jinbu (P1) and GPL (P2) using KASP marker set. The horizontal lines on each chromosome of P2 indicate sites of segments introgressed from R-donor lines. Genetic background of both was derived from Jinbu. The five R-genes were marked in the box on the chromosome of P2

Additional file 2. Table S2. List of KASP and DNA markers used in this study. Table S5. Primer sequences for qSS12 used in this study. Table S6. Primer sequences for qSS8 used in this study. Table S7. The putative ORFs in the two target regions of SS12 and SS8 based on the annotation data on Os-Nipponbare-Reference-IRGSP-1.0.

\section{Acknowledgements}

Not applicable.

\section{Authors' Contributions}

CML wrote an original draft and performed the evaluation of all the traits tested in this study, including QTLS. JPS and YCC developed the initial test population. MGB evaluated the agronomic traits of the RILs and advanced these by SSD. OYJ prepared the DNA samples for genotypic analysis and developed PCR-based markers regarding multiple resistance. HSP was involved in all aspects of the study and reviewed the manuscript and data. SJY provided critical feedback for data interpretation and contributed to the writing of the manuscript. SMK designed this study and reviewed and edited the manuscript. All the authors read and approved the final manuscript.

\section{Funding}

This work was carried out with the support of the "Cooperative Research Program for Agricultural Science and Technology Development (Project title: A study on useful breeding material resistance to environmental stresses originating from Korean native resources, Project No. PJ01480402)", Rural Development Administration, Republic of Korea.

\section{Availability of Data and Materials}

Not applicable.

\section{Declarations}

Ethics Approval and Consent to Participate

Not applicable.

\section{Consent for Publication}

Not applicable.

\section{Competing interests}

The authors declare that they have no competing interests.

\section{Author details}

${ }^{1}$ Crop Breeding Division, National Institute of Crop Science, Rural Development Administration, Wanju, Republic of Korea. ${ }^{2}$ Department of Crop Science and Biotechnology, Jeonbuk National University, Jeonju, Republic of Korea. ${ }^{3}$ Department of Ecological and Environmental System, Kyungpook National University, Sangju, Republic of Korea.

Received: 16 June 2021 Accepted: 24 November 2021

Published online: 07 December 2021

\section{References}

Bomblies K, Weigel D (2007) Hybrid necrosis: autoimmunity as a potential gene-flow barrier in plant species. Nat Rev Genet 8:382-393. https://doi. org/10.1038/nrg2082

Chattopadhyay K, Behera L, Bagchi TB et al (2019) Detection of stable QTLs for grain protein content in rice (Oryza sativa L.) employing high throughput phenotyping and genotyping platforms. Sci Rep 9:1-16. https://doi.org/ 10.1038/s41598-019-39863-2

Chen J, Ding J, Ouyang Y et al (2008) A triallelic system of S5 is a major regulator of the reproductive barrier and compatibility of indica-japonica hybrids in rice. Proc Natl Acad Sci USA 105:11436-11441. https://doi.org/ 10.1073/pnas.0804761105

Cheon KS, Baek J, II CY et al (2018) Single nucleotide polymorphism (SNP) discovery and kompetitive allele-specific pcr (KASP) marker development 
with Korean Japonica rice varieties. Plant Breed Biotechnol 6:391-403. https://doi.org/10.9787/PBB.2018.6.4.391

Chisholm ST, Coaker G, Day B, Staskawicz BJ (2006) Host-microbe interactions: shaping the evolution of the plant immune response. Cell 124:803-814. https://doi.org/10.1016/j.cell.2006.02.008

Churchill GA, Doerge RW (1994) Empirical threshold values for quantitative trait mapping. Genetics 138:963-971. https://doi.org/10.1534/genetics. 107.080101

DeYoung BJ, Innes RW (2006) Plant NBS-LRR proteins in pathogen sensing and host defense. Nat Immunol 7:1243-1249. https://doi.org/10.1038/ni1410

Fang C, Li L, He R et al (2019) Identification of S23 causing both interspecific hybrid male sterility and environment-conditioned male sterility in rice. Rice 12:1-10. https://doi.org/10.1186/s12284-019-0271-4

Ichitani K, Takemoto Y, liyama K et al (2012) Chromosomal location of HCA1 and HCA2, hybrid chlorosis genes in rice. Int J Plant Genomics. https://doi. org/10.1155/2012/649081

Ji H, Kim SR, Kim YH et al (2016) Map-based cloning and characterization of the $\mathrm{BPH} 18$ gene from wild rice conferring resistance to brown planthopper (BPH) insect pest. Sci Rep 6:1-13. https://doi.org/10.1038/srep34376

Jiang W, Chu SH, Piao R et al (2008) Fine mapping and candidate gene analysis of $h w h 1$ and $h w h 2$, a set of complementary genes controlling hybrid breakdown in rice. Theor Appl Genet 116:1117-1127. https://doi.org/10. 1007/s00122-008-0740-4

Koide Y, Ogino A, Yoshikawa T et al (2018) Lineage-specific gene acquisition or loss is involved in interspecific hybrid sterility in rice. Proc Natl Acad Sci USA 115:E1995-E1962. https://doi.org/10.1073/pnas.1711656115

Kubo T, Yoshimura A (2005) Epistasis underlying female sterility detected in hybrid breakdown in a Japonica-Indica cross of rice (Oryza sativa L.). Theor Appl Genet 110:346-355. https://doi.org/10.1007/ s00122-004-1846-y

Kubo T, Yamagata Y, Eguchi M, Yoshimura A (2008) A novel epistatic interaction at two loci causing hybrid male sterility in an inter-subspecific cross of rice (Oryza sativa L.). Genes Genet Syst 83:443-453. https://doi.org/10. 1266/ggs.83.443

Kubo T, Yoshimura A, Kurata N (2011) Hybrid male sterility in rice is due to epistatic interactions with a pollen killer locus. Genetics 189:1083-1092. https://doi.org/10.1534/genetics.111.132035

Kubo T, Takashi T, Ashikari M et al (2016) Two tightly linked genes at the hsal locus cause both F1 and F2 hybrid sterility in rice. Mol Plant 9:221-232. https://doi.org/10.1016/j.molp.2015.09.014

Li D, Chen L, Jiang L et al (2007) Fine mapping of S32(t), a new gene causing hybrid embryo sac sterility in a Chinese landrace rice (Oryza sativa L.). Theor Appl Genet 114:515-524. https://doi.org/10.1007/ s00122-006-0450-8

Long Y, Zhao L, Niu B et al (2008) Hybrid male sterility in rice controlled by interaction between divergent alleles of two adjacent genes. Proc Natl Acad Sci USA 105:18871-18876. https://doi.org/10.1073/pnas.08101 08105

Meng L, Li H, Zhang L, Wang J (2015) QTL IciMapping: integrated software for genetic linkage map construction and quantitative trait locus mapping in biparental populations. Crop J 3:269-283. https://doi.org/10.1016/j.cj. 2015.01.001

Ouyang Y (2019) Understanding and breaking down the reproductive barrier between Asian and African cultivated rice: a new start for hybrid rice breeding. Sci China Life Sci 62:1114-1116. https://doi.org/10.1007/ s11427-019-9592-6

Ouyang Y, Zhang Q (2013) Understanding reproductive isolation based on the rice model. Annu Rev Plant Biol 64:111-135. https://doi.org/10.1146/ annurev-arplant-050312-120205

Rieseberg LH, Blackman BK (2010) Speciation genes in plants. Ann Bot 106:439-455. https://doi.org/10.1093/aob/mcq126

Shen R, Wang L, Liu X et al (2017) Genomic structural variation-mediated allelic suppression causes hybrid male sterility in rice. Nat Commun. https://doi. org/10.1038/s41467-017-01400-y

Song X, Qiu SQ, Xu CG et al (2005) Genetic dissection of embryo sac fertility, pollen fertility, and their contributions to spikelet fertility of intersubspecific hybrids in rice. Theor Appl Genet 110:205-211. https://doi.org/10. 1007/s00122-004-1798-2

Suh J-P, Cho Y-C, Won Y-J et al (2015) Development of resistant genepyramided japonica rice for multiple biotic stresses using molecular marker-assisted selection. Plant Breed Biotechnol 3:333-345. https://doi. org/10.9787/pbb.2015.3.4.333

Tanksley SD, McCouch SR (1997) Seed banks and molecular maps: unlocking genetic potential from the wild. Science 277(5329):1063-1066. https:// doi.org/10.1126/science.277.5329.1063

Wang J, Liu KD, Xu CG et al (1998) The high level of wide-compatibility of variety "Dular" has a complex genetic basis. Theor Appl Genet 97:407-412. https://doi.org/10.1007/s001220050910

Widmer A, Lexer C, Cozzolino S (2009) Evolution of reproductive isolation in plants. Heredity (edinb) 102:31-38. https://doi.org/10.1038/hdy.2008.69

Xie Y, Xu P, Huang J et al (2017) Interspecific hybrid sterility in rice is mediated by OgTPR1 at the S1 locus encoding a peptidase-like protein. Mol Plant 10:1137-1140. https://doi.org/10.1016/j.molp.2017.05.005

Xie Y, Shen R, Chen L, Liu YG (2019) Molecular mechanisms of hybrid sterility in rice. Sci China Life Sci 62:737-743. https://doi.org/10.1007/ s11427-019-9531-7

Yamamoto E, Takashi T, Morinaka Y et al (2007) Interaction of two recessive genes, hbd2 and hbd3, induces hybrid breakdown in rice. Theor Appl Genet 115:187-194. https://doi.org/10.1007/s00122-007-0554-9

Yamamoto E, Takashi T, Morinaka Y et al (2010) Gain of deleterious function causes an autoimmune response and Bateson-Dobzhansky-Muller incompatibility in rice. Mol Genet Genomics 283:305-315. https://doi. org/10.1007/s00438-010-0514-y

Zhao ZG, Jiang L, Zhang WW et al (2007) Fine mapping of S31, a gene responsible for hybrid embryo-sac abortion in rice (Oryza sativa L.). Planta 226:1087-1096. https://doi.org/10.1007/s00425-007-0553-8

\section{Publisher's Note}

Springer Nature remains neutral with regard to jurisdictional claims in published maps and institutional affiliations.

\section{Submit your manuscript to a SpringerOpen ${ }^{\circ}$ journal and benefit from:}

- Convenient online submission

- Rigorous peer review

- Open access: articles freely available online

- High visibility within the field

- Retaining the copyright to your article

Submit your next manuscript at $\boldsymbol{\nabla}$ springeropen.com 